\title{
An Atypical Presentation of Chordoma: Case Report \& Review
}

\author{
*Gurnam Virdi, Iain McGraw, Elaine MacDuff, Kumar Periasamy and Himanshu Sharma \\ Orthopaedic Department, Western Infirmary Hospital, UK
}

Submission: October 25, 2017; Published: November 08, 2017

*Corresponding author: Gurnam Virdi, Clinical Research Fellow, Orthopaedic Department Level 2, Western Infirmary Hospital, Glasgow G11 6NT, United Kingdom, Email: gv1@doctors.org.uk

\begin{abstract}
Chordoma is a rare, slow-growing but locally aggressive malignant tumour for which little improvement in outcome has been reported. The management of chordoma is controversial and challenging because of their location and often large size at presentation. We report the case of a 57 year old male with radicular compression associated with an L3 chordoma that was successfully treated with a wide excision which responded well to postoperative radiotherapy. Its rare occurrence precludes controlled studies of chordoma; therefore, any response merits reporting.

Keywords: Chordoma; Lumbar spine; Tumour surgery
\end{abstract}

\section{Introduction}

Chordoma is a rare neoplasm that arises from embryonic remnants of the notochord along the axial skeleton and accounts for $1-4 \%$ of all musculoskeletal malignancies [1]. Despite histologically being considered as low-grade, chordomas are highly recurrent, radio-resistant tumours which are locally aggressive, invasive and have a poor prognosis [2]. The overall median survival time with chordoma has been estimated to be approximately 6 years from diagnosis, with a survival rate of $70 \%$ at 5 years, falling to $40 \%$ at 10 years. They have a very low incidence in patients younger than 40 years, with predominance in men between $50-60$ years of age [3].

Chordomas arise from the sacrum in approximately 50-60\% of cases, from the skull base region (spheno-occipital/nasal) in approximately $25-35 \%$ of cases, and from the mobile spine (cervical, thoracic and lumbar regions) in approximately 5\% of cases [3]. Vertebral involvement most commonly occurs in the cervical spine; the lumbar spine is least frequently affected. Chordoma has previously been considered of low metastatic potential, however distant metastasis to lung, bone, soft tissue, lymph node, liver and skin have been reported in up to $43 \%$ of patients $[4,5]$. Patient survival however seems to be less affected by distant metastasis than by local progression of chordoma [6].

The insidious course of the disease and spread along critical bony and neural structures makes clinical management of these patients difficult. The gold standard treatment for chordomas is en-bloc excision with wide margins and postoperative externalbeam radiation therapy [7]. Surgery, however, can be challenging due to tumour size, poor margination and impingement on surrounding structures. In this paper we report a rare presentation of a 57-year old male with a chordoma of the lumbar spine and we highlight current standards in diagnosis, clinical management and molecular characterization of chordomas. In addition, we outline the diagnostic and therapeutic challenges encountered.

\section{Case Report}

A 57 year-old male, with known ankylosing spondylitis, presented with an approximate one year history of right knee pain to be reviewed at our spinal clinic in August 2011. On arrival to our clinic, he confirmed mild to moderate back pain which deteriorated after activities involving spinal flexion, lifting or prolonged standing. This had been previously attributed to his ankylosing spondylitis and was consistent with his prior symptoms of this.
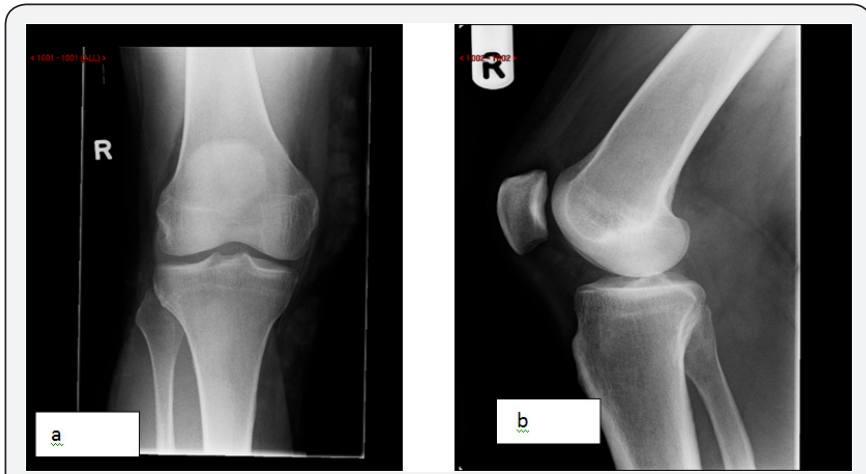

Figure 1 : Normal AP weight-bearing

a. Lateral,

b. Radiographs of right knee. Aug 2010. 


\section{Orthopedics and Rheumatology Open Access Journal}
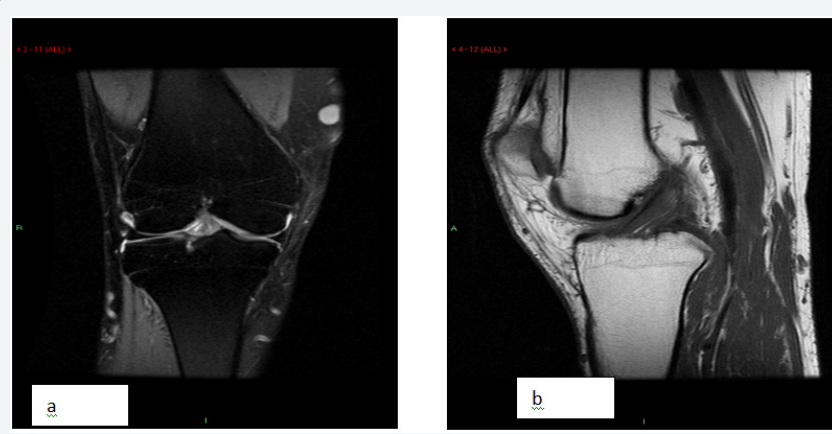

Figure 2 : Normal T2-weighted coronal MRI.

a. Showing intact menisci and T1-weighted sagittal sequence.

b. Showing intact anterior cruciate ligament. Jan 2011.

In fact, his presenting complaint was bilateral knee pain, although more prominent in the right knee, after certain activities. This was investigated with plain radiographs and later magnetic resonance imaging (MRI) scans of the right knee which were reported as normal (Figures $1 \& 2$ ). At a later visit on further questioning he complained of intermittent bilateral lower limb paraesthesia which was felt to represent the L3/4 distribution. Furthermore, he described a classic stenotic type of symptoms of claudication. Despite this, he stated having alternating good days and bad days without significant back pain. He confirmed normal bladder and bowel function and there was no history of any red flags symptoms. No abnormality was found on examination.

With his atypical presentation and past medical history, a spinal MRI scan was requested, which revealed a lesion (Figure 3). There was involvement of all three columns along with extension into the spinal canal resulting in 50\% reduction in the canal. A CT scan of the chest, abdomen and pelvis for staging did not reveal any other primary or metastatic lesion apart from a $2 \mathrm{~cm}$-sized right kidney lump detected over the upper pole of the right kidney. It was agreed at a multi-disciplinary team (MDT) meeting to seek a tissue diagnosis thus the patient was referred for image guided biopsy.
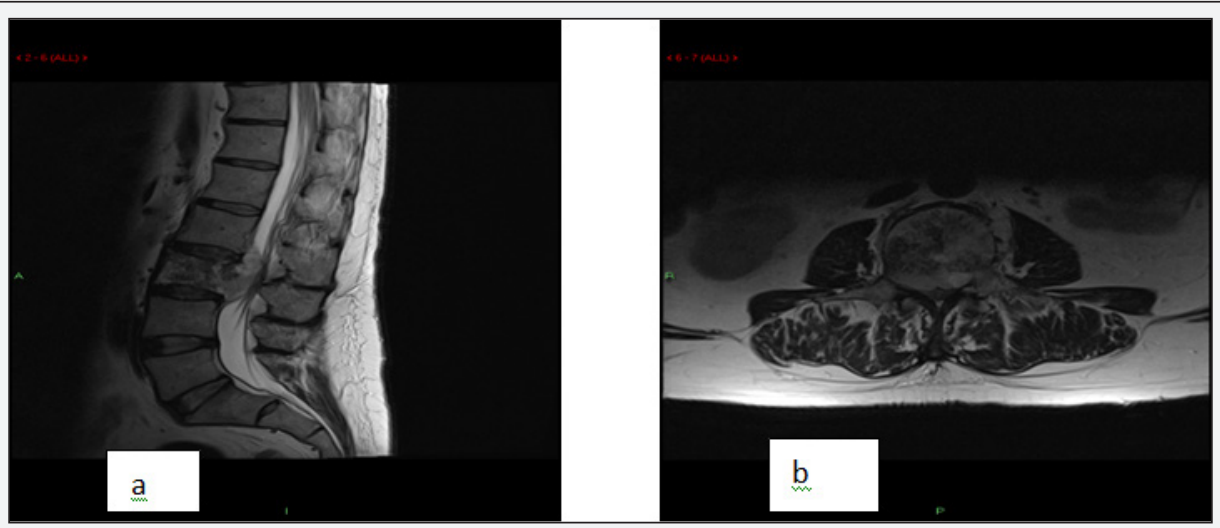

Figure 3 : Sagittal T2 sequence.

a. Demonstrating a destructive lesion with heterogenous signal extending into the spinal canal under the posterior longitudinal ligament. Axial T2 sequence.

b. Showing obliteration of the vertebral canal with complete occlusion of the right sided neural foramen at L3/4 interspace. Sep 2011.

\section{Pathological Findings}

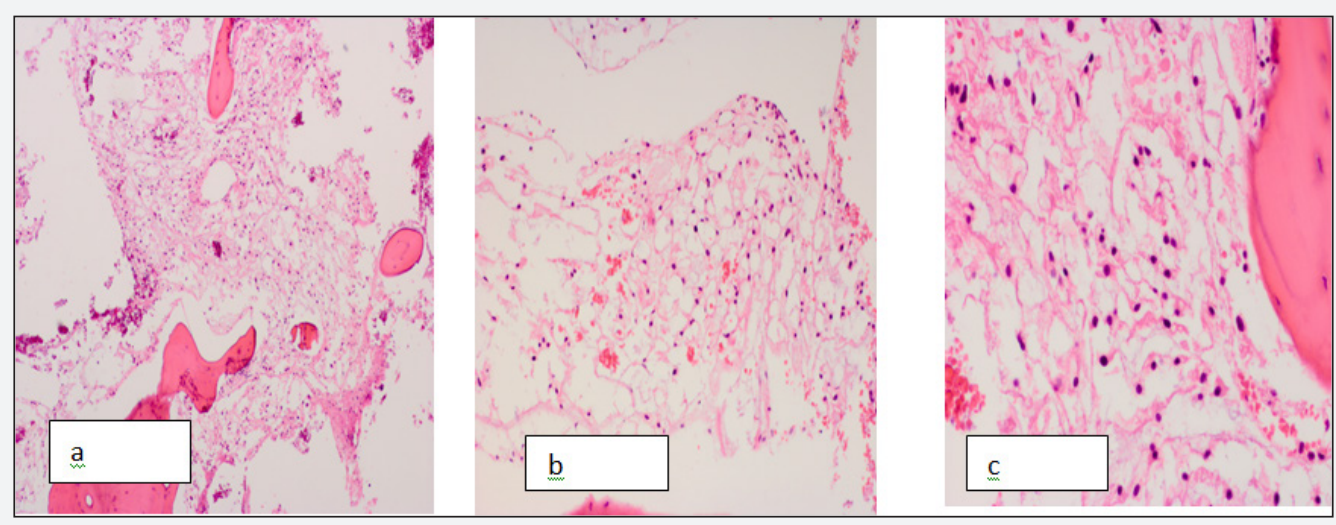

Figure 4 : Power of haemotoxylin and eosin stained images of chordoma.

a. Low.

b. Intermediate.

c. High power haemotoxylin and eosin stained images of chordoma. 
Histopathological analysis of the biopsied tissue sections showed bone within which there was a tumour composed of single cells in a myxoid/chondroid matrix. Some cells showed obvious physaliferous features. The differential diagnosis was between a chondrosarcoma and a chondroid Chordoma, and immunocytochemistry had been requested in an attempt to resolve this. Immunostaining was positive for cytokeratin AE1/1 and S100 indicating this tumour should be regarded as a chordoma rather than chondrosarcoma (Figure 4).

A soft tissue tumour biopsy was also taken which showed no evidence of chordoma at the spinous process, facet joint, lamina, ligamentum flavum, pedicle (right side) or transverse process (right side). However, there was involvement by chordoma at the superior articular process, transverse process (left side) and the pedicle (left side). A kidney needle biopsy showed that immunostains were strongly positive for renal cell carcinoma (RCC) antigen. The tumour was also positive for CD10, vimentin, AE1/3, ENA, NSE and S100. It was negative for HBME1. The immunoprofile confirmed the diagnosis of a primary clear cell renal cell carcinoma, although it also confirmed that the previous LV3 biopsy slides showed a chordoma affecting L3 vertebra rather than a metastatic RCC. The aim of surgical intervention was to de-bulk the tumour by a two stage procedure. The first stage involved D12 to L5 posterior spine instrumentation and posterior L3 vertebrectomy. The second stage involved an anterior lumbar L2 and L3 corpectomy and cage reconstruction. The patient underwent postoperative radiotherapy and was asymptomatic at both 6 and 12 month follow-ups (Figure 5).

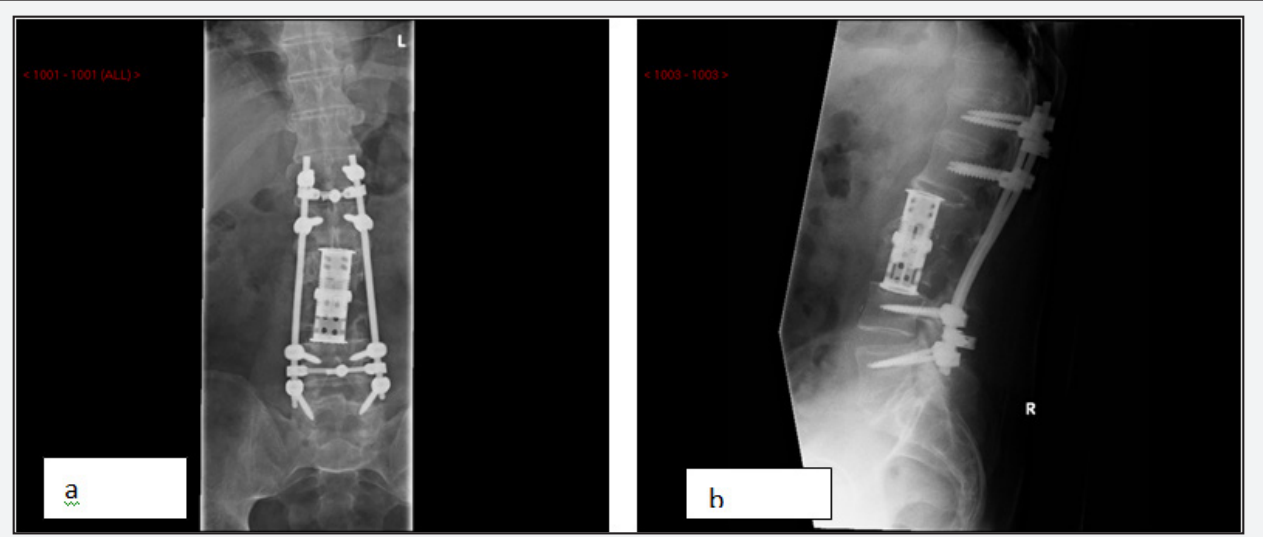

Figure 5 : Post-operative radiographs. Nov 2011.

\section{Discussion}

Chordomas were first characterised microscopically by Virchow in 1857 [8]. He described these tumours to be made up of unique 'physaliferous' cells consisting of intracellular 'bubbly' vacuoles. These physaliferous features of chordoma remain a distinguishing feature. Virchow hypothesised that chordomas were derived from undifferentiated notochordal remnants that reside within the vertebral bodies throughout the axial skeleton. In fact, the most compelling evidence to support this hypothesis was role of the brachyury gene. Brachyury regulates several stem-cell genes and has been implicated in promoting epithelial-mesenchymal transition in other human carcinomas. It is still unclear what role brachyury has in the pathogenesis of chordomas, although it has been identified duplicating and remarkably over-expressed in chordoma biopsy sample analysis [9-11]. In addition to support this hypothesis, examination of human embryos and foetuses showed that notochordal cell nests correspond and distribute to the sites of occurrence of chordoma [12].

The slow-growing nature of chordomas means they are often clinically silent until the later stages of disease progression. The clinical manifestations vary and depend on the location of the tumour and any impingement upon adjacent structures. Chordomas at the base of the skull often grow in the clivus and can present typically with cranial-nerve palsies, visual disturbances and headache or rarely with intracranial haemorrhage, epistaxis or CSF rhinorrhea [13]. Chordomas of the mobile spine and sacrum can remain asymptomatic for a long time and/or present with a variety of non-specific symptoms such as localised back pain, paraesthesia, and bladder or bowel dysfunction. In addition, there may be signs of radiculopathies related to the spinal level at which they occur [14]. Cervical chordomas can present as an oropharyngeal mass, with dysphagia or even with airway obstruction.

Staging is accomplished by a thorough history and physical examination, followed by a combination of diagnostic tests including CT, MRI and bone scans. Initially, the presentation of chordoma on CT scan is of bone destruction centred in the vertebral body, with an associated anteriorly or laterally situated, paraspinal soft-tissue mass that may contain calcification. Following vertebral body involvement, the pedicles, laminae, and spinal process may then become involved; adjacent intervertebral disc spaces are usually spared. At present, MRI scanning (with gadolinium contrast) is the method of choice for the diagnosis and preoperative assessment of chordomas as it is extremely sensitive in detecting soft tissue spread and tumour recurrence. Spinal chordomas show calcification and bony expansion and display features that appear isointense or hypointense on T1-weighted 


\section{Orthopedics and Rheumatology Open Access Journal}

MRI images, hyperintense on T2-weighted MRI images, and enhance with gadolinium [15]. Bone scans have also been utilised in diagnosis of chordomas, however, they can be normal.

Fine needle aspiration biopsy (or core needle biopsy) is a widely accepted technique for rapid, accurate and economical diagnosis of a wide variety of lesions. The histologic and gross features of chordomas are well described in the literature. Chordomas exhibit various degrees of histological atypia and usually manifest as one of three histological variants: conventional, chondroid, or dedifferentiated [16]. Conventional chordomas are the most common type and appear as soft, grayish, lobulated tumours composed of groups of cells in a pale myxoid matrix separated by fibrous connective tissue septa. They have round nuclei and an abundant, vacuolated cytoplasm described as 'physaliferous'. The tumour is usually surrounded by pseudocapsule however, extension into surrounding bone and soft tissue can be seen. Chondroid chordomas histologically show features of both chordoma and chondrosarcoma (a malignant cartilage-forming tumour) [16].

Some controversy exists regarding the histopathology of these tumours. Classically, chordomas were pathologically identified by their physaliferous features and immunoreactivity for S-100 and epithelial markers such as epithelial membrane antigen (MUC1) and cytokeratins $[17,18]$. However, both chondroid chordomas and chondrosarcomas share their S-100 immunoreactivity, making it challenging to distinguish between the two [19]. Accurate diagnosis of tumours of the spine and skull base is prognostically important. Chordomas and chondrosarcomas have sufficient morphological overlap yet both represent two biologically distinct categories of mesenchymal neoplasms [19]. Some studies have suggested that the notochord developmental transcription factor, brachyury, could be a discriminating biomarker for chordomas $[9,19,20]$. In fact, when combined with cytokeratin staining, sensitivity and specificity for detection of chordoma was $98 \%$ and $100 \%$, respectively [19]. Dedifferentiated chordomas are rapidly growing tumours which are biphasic, with areas of high grade sarcoma that co-exist alongside conventional or chondroid chordomas.

Surgical resection, radiation therapy, and chemotherapy are the current proposed therapy modalities for the management of spinal chordomas. Due to the delay in presentation of clinical symptoms, the tumour has potential to reach an extensive size, thus making surgical management a challenge to the surgeon. Wide en-bloc surgical resection remains the mainstay of management of chordomas. The surgical techniques for marginfree en-bloc tumour resection have been proven to be effective in terms of local control and long term prognosis for chordoma occurring in the mobile spine [21-23]. One study suggested that contamination of the surgical wound via cell seeding is responsible for recurrence, and therefore advocate complete excision of the tumour during initial surgery [24]. Surgical outcome is dependent on location of tumour and the degree of spread and bulky tumours near important adjacent structures may preclude negative margin resections.
Chordomas are considered radio-resistant tumours and require extreme doses of radiation which cannot be delivered safely because it would exceed the tolerance of most neurological structures, especially the brainstem and the optic pathway [25]. There is some consensus that radiation therapy in combination with surgery provides an added advantage. Advances in radiation technology with the introduction of hadrons (high dose protons) have led to higher doses of radiation being delivered to the target with minimal injury to surrounding tissue [26]. Treatment with conventional radiation therapy at doses of 40-60 Gy has led to 5 -year local control of only $10-40 \%[27,28]$.

Studies exploiting the use of hadron therapy in chordomas, especially at the skull base and cervical spine show local control at 5 years of $50-60 \%$ [4]. Preliminary evidence suggests that hadron therapy is a more promising strategy, as opposed to photon therapy, when coupled with surgery [29]. Proton-beam therapy with wide en-bloc excision is the accepted treatment standard in the management of chordomas in many centres, especially in patients with a primary tumour as opposed to recurrence of disease. Chordomas are not reported to be sensitive to chemotherapy; however responses have been reported in patients with highgrade dedifferentiated chordomas [30].

\section{Diagnostic Challenges}

This case report is interesting on several accounts. The patient presented as an atypical case with bilateral knee pain in his late fifties. There was minimal knee abnormalities e.g. valgus deformity and swelling. Initially, we thought the symptoms were due to osteoporosis compression fractures, however this can be distinguished from bone tumours as the symptoms of the latter tend not to improve. His back pain differed little from that preexisting due to his ankylosing spondylitis and it took almost one year to diagnose a lumbar chordoma. The delay in diagnosis was due to the absence of red flag symptoms and thinking it was pain associated with ankylosing spondylosis.

Because of their location, lumbar chordomas may present with radicular symptoms and may easily be confused with more common tumours in the lumbar spine such as aneurysmal bone cyst, giant cell tumour, haemangioma, myeloma and metastasis. Thus, preoperative diagnosis can be difficult. Secondary bone tumours are more common than primary bone tumours; hence it is important to have a high suspicion, as in this case, of a primary bone tumour since there were no other vertebral issues or systemic symptoms. It is important to carry out a biopsy and histopathological examination - tasks which can prove to be a diagnostic challenge.

\section{Therapeutic Challenges}

The tumour had invaded both anterior and posterior aspects of the involved vertebrae which then subsequently required the spine to be stabilized and the affected vertebrae removed. Stabilizing the spine requires the use of spinal radiographs once the patient is put into a certain position. This was challenging in our patient as he 
has kyphosis from ankylosing spondylosis. Thus, the difficulty of tumour removal in ankylosing spondylosis can potentially pose positional problems on the table. We overcame this by the use of cushions.

Removal of the tumour is technically demanding - removing the posterior tumour took approximately 8 hours, thus we recommend sharing the workload between two surgeons. In addition, we recommend a one week waiting period before operating on the anterior tumour as this procedure lasts a further 8-10 hours. Furthermore, we do not advise operating after a few days since the patient would still be undergoing metabolic response from surgery. It is appropriate to summon a vascular surgeon in case the tumour is in adherence to large vessels. We found that we had to remove one and half vertebrates instead of one, hence it is important to make sure blood and blood products are readily available as there may be potential large losses of blood.

Once the spine is fixed in such patients, we carry out radiotherapy once the tumour has been resected. We used bone grafts; however this has a limitation as radiotherapy can destroy this. Only two centres' in the world offer polyaryletherketones (PEEK) implant and so there is a need to apply for funding. The question arises about who should be doing these cases, i.e. primary bone tumours. Currently, there are only 3 centres in the UK that do this - Glasgow, Birmingham and London.

\section{Conclusion}

The lumbar spine is an uncommon location for chordoma. This case report demonstrates the diagnostic and therapeutic difficulties they may present throughout the patient journey. Surgery is the primary modality to achieve the best long-term control. However, the location of these tumours makes en bloc excision to achieve adequate negative margins technically challenging. Conventional radiotherapy has a theoretical role, however, the high doses required for these radio-resistant tumours lead to significant toxicity to surrounding normal tissues and limit its therapeutic value. Newer techniques and charged particle radiotherapy allow for better dose delivery, and hence better disease control. Cytotoxic chemotherapy has virtually no role in this disease however; molecularly targeted therapy is showing significant promise and is an area of great potential.

\section{References}

1. Jemal A, Siegel R, Ward E, Murray T, Xu J, et al. (2007) Cancer statistics. CA Cancer J Clin 57(1): 43-66.

2. P Bergh, LG Kindblom, B Gunterberg, F Remotti, W Ryd, et al. (2000) Prognostic factors in chordoma of the sacrum and mobile spine: a study of 39 patients. Cancer 88(9): 2122-2134.

3. ML McMaster, AM Goldstein, CM Bromley, N Ishibe, DM Parry (2001) Chordoma: incidence and survival patterns in the United States, 19731995. Cancer Causes Control 12(1): 1-11.

4. Catton C, O'Sullivan B, Bell R, laperriere N, Cummings B, et al. (1996) Chordoma: Long-term follow-up after radical photon irradiation. Radiother Oncol 41(1): 67-72.
5. Chambers PW, Schwinn CP (1979) Chordoma. A clinicopathologic study of metastasis. Am J Clin Pathol 72(5): 765-776.

6. S Boriani, F Chevalley, JN Weinstein, R Biagini, L Campanacci, et al. (1996) Chordoma of the spine above the sacrum. Treatment and outcome in 21 cases. Spine 21(13): 1569-1577.

7. Tai PT, Craighead P, Liem SK, Jo BH, Stitt L, et al. (2000) Management issues in chordoma: A case series. Clin Oncol (R Coll Radiol) 12(2): 8086.

8. Mark YS Soo (2001) Chordoma review of clinicoradiological features and factors affecting survival. Australas Radiol 45(4): 427-434.

9. J Shen, CD Li, HL Yang, J Lu, TM Zou, et al. (2011) Classic chordoma coexisting with benign notochordal cell rest demonstrating different immunohistological expression patterns of brachyury and galectin-3. J Clin Neurosci 18(1): 96-99.

10.XR Yang, D Ng, DA Alcorta, NJ Liebsch, E Sheridan, et al. (2009) T (brachyury) gene duplication confers major susceptibility to familial chordoma. Nat Genet 41(11): 1176-1178.

11. Henderson SR, Guiliano D, Presneau N, McLean S, Frow R, et al. (2005) A molecular map of mesenchymal tumors. Genome Biol 6(9): R76.

12.JR Salisbury, MH Deverell, MJ Cookson, WF Whimster (1993) Threedimensional reconstruction of human embryonic notochords: clue to the pathogenesis of chordoma. J Pathol 171(1): 59-62.

13. St Martin M, Levine SC (2003) Chordomas of the skull base: manifestations and management. Curr Opin Otolaryngol Head Neck Surg 11(5): 324-327.

14. P Bergh, LG Kindblom, B Gunterberg, F Remotti, W Ryd, et al. (2000) Prognostic factors in chordoma of the sacrum and mobile spine: a study of 39 patients. Cancer 88 (9): 2122-2134.

15. Stephens GC, Schwartz HS (1993) Lumbosacral chordoma resection: image integration and surgical planning. J Surg Oncol Dec 54(4): 226232.

16. R Chugh, H Tawbi, DR Lucas, JS Biermann, SM Schuetze, et al. (2007) Chordoma: the nonsarcoma primary bone tumor. Oncologist 12(11): 1344-1350.

17. JP Crapanzano, SZ Ali, MS Ginsberg, MF Zakowski (2001) Chordoma: a cytologic study with histologic and radiologic correlation. Cancer 93(1): 40-51.

18. A Mitchell, BW Scheithauer, KK Unni, PJ Forsyth, LE Wold, et al. (1993) Chordoma and chondroid neoplasms of the spheno-occiput. An immunohistochemical study of 41 cases with prognostic and nosologic implications. Cancer 72(10): 2943-2949.

19. GJ Oakley, K Fuhrer, RR Seethala (2008) Brachyury, SOX-9, and podoplanin, new markers in the skull base chordoma vs chondrosarcoma differential: a tissue microarray-based comparative analysis. Mod Pathol 21(12): 1461-1469.

20. AR Sangoi, J Karamchandani, B Lane, JP Higgins, RV Rouse, et al. (2011) Specificity of brachyury in the distinction of chordoma from clear cell renal cell carcinoma and germ cell tumors: a study of 305 cases. Mod Pathol 24(3): 425-429.

21. JJ Bosma, TJ Pigott, BH Pennie, DC Jaffray (2001) En bloc removal of the lower lumbar vertebral body for chordoma. Report of two cases. J Neurosurg 94(2): 284-291.

22. KY Hsu, JF Zucherman, N Mortensen, JO Johnston, J Gartland (2000) Follow-up evaluation of resected lumbar vertebral chordoma over 11 years: A case report. Spine 25(19): 2537-2540.

23. N Sundaresan, AA Steinberger, F Moore, VP Sachdev, G Krol, et al. (1996) Indications and results of combined anterior-posterior approaches for spine tumor surgery. J Neurosurg 85(3): 438-446. 
24. Kaiser TE, Pritchard DJ, Unni KK (1984) Clinicopathologic study of sacrococcygeal chordoma. Cancer 53(11): 2574-2578.

25. J Debus, EB Hug, NJ Liebsch, D O’Farrel, D Finkelstein, et al. (1997) Brainstem tolerance to conformal radiotherapy of skull base tumors. Int J Radiat Oncol Biol Phys 39(5): 967-975.

26. M Austin-Seymour, J Munzenrider, R Linggood, M Goitein, L Verhey, et al. (1990) Fractionated proton radiation therapy of cranial and intracranial tumors. Am J Clin Oncol 13(4): 327-330.

27. PA Forsyth, TL Cascino, EG Shaw, BW Scheithauer, JR O’Fallo, et al. (1993) Intracranial chordomas: a clinicopathological and prognostic study of 51 cases. J Neurosurg 78(5): 741-747.
28. G Noël, L Feuvret, V Calugaru, H Mammar, C Haie-Meder, et al. (2005) Chordomas of the base of the skull and upper cervical spine. One hundred patients irradiated by a 3D conformal technique combining photon and proton beams. Acta Oncol 44(7): 700-708.

29. Goitein M, Cox JD (2008) Should randomized clinical trials be required for proton radiotherapy? J Clin Oncol 26(2): 175-176.

30. GF Fleming, PS Heimann, JK Stephens, MA Simon, MK Ferguson, et al. (1993) Dedifferentiated chordoma. Response to aggressive chemotherapy in two cases. Cancer 72(3): 714-718.

\section{Your next submission with Juniper Publishers will reach you the below assets}

- Quality Editorial service

- Swift Peer Review

- Reprints availability

- E-prints Service

- Manuscript Podcast for convenient understanding

- Global attainment for your research

- Manuscript accessibility in different formats

( Pdf, E-pub, Full Text, Audio)

- Unceasing customer service

Track the below URL for one-step submission https://juniperpublishers.com/online-submission.php 